\title{
Variación florística de especies arbóreas a escala local en un bosque de tierra firme en la Amazonia colombiana
}

\author{
Juan Sebastian Barreto SILVA Álvaro Javier Duque MONTOYA², Dairon Cárdenas LÓPEZ³, Flavio \\ Humberto Moreno HURTADO ${ }^{4}$
}

\begin{abstract}
RESUMEN
El presente estudio se llevó a cabo en cinco hectáreas de una parcela permanente establecida en el Parque Nacional Amacayacu, Amazonia colombiana. En éste, se evaluó el efecto de la variación ambiental y la configuración espacial sobre los patrones florísticos de las especies arbóreas $(\mathrm{DAP}>10 \mathrm{~cm})$ a escala local en un bosque de tierra firme. Se estudió la variación florística y ambiental en cuadrantes de 20x20 m. Adicionalmente, se consideraron diferentes categorías de abundancia (total, alta, media y baja). Se utilizó el Análisis de Correspondencia Linealizado y el Análisis de Correspondencia Canónica, seguido de una partición de la variación, para cuantificar la magnitud a la cual el ambiente y la limitación en dispersión determinan la variación florística. La fracción espacial, representando procesos de autocorrelación como la limitación en dispersión, se analizó mediante dos métodos: Asumiendo un polinomio de tercer grado y por el método de Coordenadas Principales de Matrices Vecinas (PCNM). La diversidad beta de la parcela fue baja. El PCNM aparece como el método de análisis más apropiado para estudios a esta escala. Las diferencias florísticas explicadas a lo largo de la parcela de 5-ha fueron principalmente asociadas con procesos biológicos como la limitación en dispersión. La mayor parte de la variación florística, no obstante, no fue explicada por las variables ambientales o espaciales consideradas. En conclusión, estos resultados sugieren que procesos aleatorios son determinantes esenciales de la variación espacial de las especies arbóreas a escala local en tierra firme en los bosques en el Parque Nacional Amacayacu.
\end{abstract}

PALABRAS-CLAVE: abundancia, análisis espacial, diversidad beta, distribución de especies, rareza

\section{Floristic variation of canopy tree species at a local scale on tierra firme forests in colombian Amazonia}

\begin{abstract}
SUMMARY
This study was carried out in a 5-ha permanent plot established in the Amacayacu National Park, Colombian Amazonia. We assessed the extent at which floristic patterns of tree species were determined by either the environmental variation or the spatial configuration in tierra firme forests. Floristic and environmental variation was assessed on quadrants of $20 \times 20 \mathrm{~m}$. To run the analysis, four different categories of abundance (all, high, medium and low) were considered. We used Detrended Correspondence Analysis and Canonical Correspondence Analysis, followed by a variation partitioning, to analyze the patterns of species distribution in order to quantify the role played by both environmental filtering and dispersal limitation. The spatial template, which represents dispersal limitation, was evaluated in two different ways: using a third degree polynomial and using the Principal Coordinates of Neighbour Matrix (PCNM) method. Beta diversity was relatively low within the plot. PCNM appears as the most appropriate method to analyze the spatial template at this scale. The floristic differences explained along the 5-ha plot were mainly associated to biological processes, such as dispersal limitation. The largest proportion of community variation in our dataset was unexplained by either environmental or spatial data. In conclusion, these results support random processes as the major drivers of the spatial variation of tree species at a local scale on tierra firme forests in the Amacayacu National Park.
\end{abstract}

KEYWORDS: abundance, spatial analysis, beta diversity, species distribution, rarity.

\footnotetext{
1 Universidad Nacional de Colombia Sede Medellín. E-mail: sbarreto@unal.edu.co

2 Universidad Nacional de Colombia Sede Medellín. E-mail: ajduque@unal.edu.co

3 Instituto Amazónico de Investigaciones Científicas SINCHI. E-mail: dcardenas@sinchi.org.co

${ }^{4}$ Universidad Nacional de Colombia. E-mail: fhmoreno@unal.edu.co
} 


\section{INTRODUCCION}

Los patrones de distribución de las especies son un tema central de la teoría ecológica, los cuales cobran una mayor relevancia en los bosques tropicales debido su alta complejidad (Gentry 1988; He et al. 1996; Condit et al. 2000). La vegetación determinada de una zona, es el resultado proveniente de la interacción entre los factores ambientales, los procesos biológicos y un conjunto de especies que cohabitan un espacio continuo (Matteucci 1982). La forma como se distribuyen los organismos en ese espacio geográfico es crucial, ya que permite inferir acerca del uso de los recursos por las especies y refleja el efecto de la adaptación a las condiciones del hábitat y/o de la limitación en dispersión sobre la estructura de las comunidades (Condit et al. 2002; Tuomisto et al. 2003).

De acuerdo con la teoría de nicho se asume que las especies coexisten como respuesta a adaptaciones específicas con respecto a las condiciones del hábitat, las cuales les otorgan una ventaja competitiva frente a otras especies (Hutchinson 1959; Tilman y Pacala 1993). En este sentido, hábitat se refiere a discontinuidades medioambientales representadas por la heterogeneidad topográfica, el clima, el material parental o los suelos, entre otros (Rees et al. 2001; Pyke et al. 2001; Tuomisto et al. 2003). Se ha reportado además que la respuesta a la variación ambiental en muchos casos es diferencial de acuerdo con el grado de desarrollo de los individuos, siendo mayor en los estadios juveniles (Svenning 1999; Harms et al. 2001; Duque et al. 2002; Comita et al. 2007; Zillio y Condit 2007).

Contrario a lo anterior, la teoría neutral propone que procesos como la limitación en dispersión y eventos estocásticos de especiación y extinción, determinan los ensambles de especies de las comunidades arbóreas (Hubbell et al. 2001; He y Hu 2005; Hubbell 2005; Zillio y Condit 2007). La neutralidad per se, lo que define es la equivalencia ecológica en términos competitivos de todos los individuos de la comunidad. De esta manera, los eventos de natalidad, mortalidad y ocupación del espacio geográfico de las especies dentro del bosque, pueden considerarse básicamente un asunto de simple y pura probabilidad (Hubbell 1979,2001).

Los patrones y procesos ecológicos resultantes de los estudios dependen además de la escala espacial a la cual se examinan (He et al. 2002). A escala regional e intermedia, en la cuenca amazónica, parece existir consenso acerca del efecto sobre la composición florística y distribución de las comunidades de plantas producto de las diferencias ambientales a escala de ecosistemas y paisajes (Gentry 1988; Duivenoorden y Lips 1995; Duivenvoorden y Duque in press; Tuomisto et al. 1995,2003; Duque et al. 2002). Sin embargo, a escala local $\left(<1 \mathrm{Km}^{2}\right)$, en los bosques tropicales, el efecto de la variación ambiental sobre la estructura de las comunidades arbóreas, es aún motivo de debate (Duque et al. 2003; Valencia et al. 2004; Poulsen et al. 2006; John et al. 2007).

De un lado, hay quienes soportan que en paisajes como tierra firme, donde el gradiente ambiental disminuye, a escala local el efecto de los suelos sobre los patrones de distribución de las especies es mínimo, recayendo la mayor explicación en procesos aleatorios o biológicos como la limitación en dispersión (Duque et al. 2003; He et al. 2002; Valencia et al. 2004). En contraste, un mayor efecto de la variación edáfica y topográfica sobre la composición florística, ha sido reportada en estudios con diferentes tipos de plantas en bosque tropicales (Poulsen et al. 2006; John et al. 2007).

El estudio de la distribución de especies de acuerdo con categorías de abundancia, además de indagar acerca de los efectos del tamaño de la muestra, busca explicar si la relación de éstas con su medioambiente, está reflejada en la distribución de las abundancias relativas (Brown et al. 1995; Alonso et al. 2008). En otras palabras, pretende indagar acerca de la diferencia adaptativa que supone ventajas competitivas de algunas especies respecto al uso de los recursos en un hábitat determinado (Preston 1962; Tilman y Pacala 1993; Brown et al. 1995). Según Preston (1962), las especies de abundancia intermedia pueden ser especialmente útiles como indicadores diferenciales de la adaptación al nicho. Este tipo de preguntas resultan relevantes especialmente en los bosques tropicales donde muy pocas especies están representadas por un alto porcentaje de individuos (Pitman et al. 2001), mientras la mayoría de ellas tienden generalmente a ser localmente raras (He et al. 1996; Condit et al. 2000; Hubbell 2001; He et al. 2002; McGill et al. 2007; Zillio y Condit 2007).

Esta investigación tiene como objetivo evaluar la distribución de especies arbóreas del dosel en tierra firme, a escala local, en el Parque Nacional Natural Amacayacu, y su posible relación con la variación de algunas propiedades topográficas y edáficas, y/o procesos biológicos espacialmente estructurados tales como la limitación en dispersión. De igual manera, se busca entender si dicha relación puede variar de acuerdo con diferentes categorías de abundancia. Se espera aportar herramientas de manejo que fortalezcan los modelos de conservación de los bosques en la cuenca Amazónica.

\section{MÉTODOS}

\section{ÁREA DE ESTUDIO}

El área de estudio se localiza al extremo sur de Colombia, en el Parque Nacional Natural Amacayacu (-3 $3^{\circ}$ ' Latitud Sur $70^{\circ} 19^{\prime}$ Longitud Oeste), en bosques de tierra firme. La zona de vida según la clasificación de Holdridge (1979), es bosque húmedo tropical: Bh-T. Las 5 hectáreas de bosque seleccionadas se ubican en una Parcela Permanente establecida para el estudio y monitoreo de la vegetación de tierra firme en 
el parque. Cada hectárea es un rectángulo o faja de 20x500 $\mathrm{m}$, que se divide en 25 cuadrantes adyacentes de $20 \times 20 \mathrm{~m}$. Este estudio utilizó 125 cuadrantes o unidades muestrales, comprendidas entre las hectáreas A y E, en un rectángulo de 100x500 m (Figura 1).

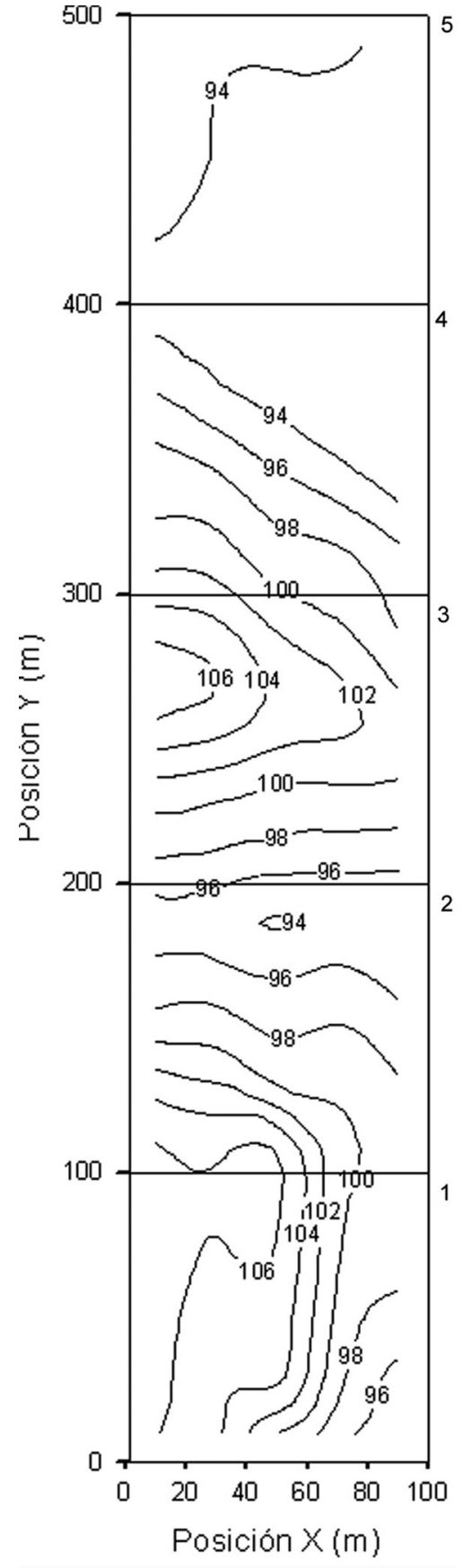

Figura 1 - Altimetría 5-ha Parcela Amacayacu TRABAJO DE CAMPO

En las primeras 5 hectáreas arriba mencionadas se marcaron, codificaron, midieron y colectaron todos los árboles, helechos arbóreos y palmas con diámetro mayor o igual a $10 \mathrm{~cm}$ a $1,3 \mathrm{~m}$ de altura (DAP) siguiendo la metodología del CTFS (Condit 1998). La colección botánica la realizaron equipos de trabajo conformados por investigadores del Instituto Sinchi, la Universidad Nacional de Colombia y coinvestigadores de la comunidad indígena local de Palmeras. Se colectaron 1703 muestras botánicas que fueron identificadas a especie, y sirvieron para homologar al resto de individuos. Este material botánico se procesó y determinó en el Herbario Amazónico Colombiano COAH del Instituto Sinchi.

En cada cuadrante se tomaron tres muestras de suelo del primer horizonte mineral, una cada cinco metros en la línea central del cuadrante $(5,10$ y $15 \mathrm{~m})$ y entre 0 y 10 $\mathrm{cm}$. de profundidad, las cuales se mezclaron para completar un kilogramo por cuadrante. Los análisis de las propiedades físico-químicas del suelo se realizaron en el Laboratorio de Ecología "César Pérez Figueroa" adscrito al Departamento de Ciencias Forestales de la Universidad Nacional de Colombia, sede Medellín. Se analizó el contenido extraíble o disponible en los suelos de $\mathrm{pH}$, materia orgánica, iones intercambiables $(\mathrm{Ca}, \mathrm{Mg}, \mathrm{K}, \mathrm{Al}$ y $\mathrm{P})$, así como la textura representada en los porcentajes de arena, limo y arcilla (A, L y Ar, respectivamente). Las variables topográficas consideradas fueron: Altitud, pendiente y curvatura, definidas de acuerdo a la metodología utilizada por Harms et al. (2001) y por Valencia et al. (2004).

\section{ANÁLISIS DE LOS DATOS}

Las abundancias de las especies se agruparon en octavas (Preston 1948) y posteriormente se clasificaron en categorías de abundancia: Alta (>16 Individuos), media (5-16) y baja (1-4). Sin embargo no se consideró en el análisis a las especies con un solo individuo con el fin de disminuir el ruido proveniente de la gran cantidad de especies raras. Por lo tanto, se evaluó la variabilidad florística sólo con las especies que estuvieran representadas al menos con 2 individuos en las cinco hectáreas. La distribución de especies se analizó empleando métodos de ordenación (gradiente) directos e indirectos (Jongman et al 1987), mediante el programa estadístico CANOCO versión 4.52 (ter Braak \& Smilauer 2002).

El análisis de correspondencia linealizado (DCA en inglés), definido como análisis de gradiente indirecto, asume el modelo Gaussiano como modelo de respuesta de las especies (ter Braak et al. 1987), y permite evaluar la diversidad beta en términos de desviaciones estándar entre unidades muestrales. En éste tipo de análisis, se asume que unidades muestrales más cercanas entre si en el diagrama de ordenación, tienen mayor similaridad florística. Como método directo de ordenación se empleó el análisis de correspondencia canónico (CCA en inglés), que permite correlacionar la distribución de especies con factores medioambientales. Las variables ambientales incluidas, referidas principalmente al 
contenido de minerales y la topografía del terreno fueron logarítmicamente transformadas. Para complementar el CCA, se incluyeron variables espaciales de acuerdo a la posición geográfica referida a un plano cartesiano de las unidades muestrales. El espacio geográfico, comúnmente asociado con el efecto de la dispersión (Jones et al. 2008 y citas referidas), fue analizado por medio de dos métodos diferentes: 1) usando un polinomio de tercer grado (Borcard et al. 1992); las variables que componen dicho polinomio son: $\mathrm{x}, \mathrm{y}, \mathrm{xy}, \mathrm{x}^{2}, \mathrm{y}^{2}, \mathrm{x}^{2} \mathrm{y}, \mathrm{xy}^{2}$, $x^{3} y^{3}{ }^{3}$. 2) Usando el método del análisis de Coordenadas Principales de Matrices Vecinas (PCNM, en Inglés); se uso como umbral para generar la matriz truncada una distancia de $20 \mathrm{~m}$, y reemplazando todas las distancias mayores por 4 veces esta distancia, es decir $80 \mathrm{~m}$ (Borcard y Legendre 2002). Todos estos análisis fueron llevados a cabo para todos los datos de especies y para cada una de las categorías de abundancia. Las variables polinomiales representan estructuras lineales o curvas de dimensión similar al área de estudio, mientras que las de PCNM consisten en ondas ortogonales, cuyo rango de longitud de onda comprende todas las escalas definidas por el muestreo (Jones et al. 2008).

Para hacer la selección de las variables ambientales y espaciales incluidas en la partición de la variación se corrieron CCAs independientes para cada grupo de variables (ambientales o espaciales) y para cada categoría de abundancia. Para la selección de variables ambientales se llevó a cabo una selección manual asumiendo una probabilidad de 0.05 para la retención de cada variable independiente; para las variables ambientales se hizo la transformación logarítmica, con base en 199 permutaciones de los datos. Para las variables espaciales se siguió el mismo proceso sin hacer transformación logarítmica de los datos. Aquellas variables ambientales y espaciales escogidas independientemente fueron unidas para analizar el total de variación explicado de los datos de composición florística en la parcela.

Finalmente, y con base en el CCA, se realizó una partición de la variación de los diferentes componentes para calcular el porcentaje explicado por los datos ambientales, los datos espaciales, o ambos (Borcard et al. 1992). Se analizaron por separado ambos métodos espaciales, con el fin de comparar y entender mejor la capacidad explicatoria de cada uno de ellos (Jones et al. 2008). Este análisis se llevo a cabo de nuevo para todos los datos y para cada una de las categorías de abundancia.

\section{RESULTADOS}

\section{SUELOS}

En general el análisis de las propiedades físicas y químicas de los suelos de las 125 muestras (Tabla 1), determina que son suelos ácidos de acuerdo al $\mathrm{pH}(3.75-4.88)$ у а las concentraciones de Aluminio $(0.30-1.00 \mathrm{cmol}(+) / \mathrm{Kg})$. La capacidad de intercambio catiónico efectiva $(\mathrm{CICE}=$ $\mathrm{Al}+\mathrm{Ca}+\mathrm{Mg}+\mathrm{K})$, varia en los 125 cuadrantes de 1.69 a 8.33 $\mathrm{cmol}(+) / \mathrm{Kg}$, solamente, la concentración de Aluminio conforma el $33.1 \%$ en promedio de la CICE, mientras que la concentración de Calcio y Magnesio juntas, representan el 54\% de esta (Tabla 2). Los suelos tienen una fracción alta de grano fino (arcilla $25 \%$ y limo $45 \%$ ), con arenas del 30\% en promedio.

Tabla 1 - Análisis de propiedades físico-químicas de los suelos.

\begin{tabular}{lcccc}
\hline Variable & Promedio & Rango & $\begin{array}{c}\text { Desviación } \\
\text { Estándar }\end{array}$ & Coeficientevariación \\
\hline pH & 4.30 & $3.75-4.88$ & 0.27 & $6 \%$ \\
Materia Orgánica (\%) & 3.13 & $1.36-6.12$ & 0.74 & $24 \%$ \\
Aluminio (cmol(+)/ & 0.70 & $0.30-1.00$ & 0.17 & $24 \%$ \\
Kg) & & & & $96 \%$ \\
Calcio (cmol(+)/Kg) & 0.90 & $0.12-6.22$ & 0.86 & $64 \%$ \\
Magnesio $(\mathrm{cmol}(+) / \mathrm{Kg})$ & 0.53 & $0.17-2.44$ & 0.34 & $39 \%$ \\
Potasio (cmol $(+) / \mathrm{Kg})$ & 0.28 & $0.16-0.56$ & 0.11 & $46 \%$ \\
CICE (cmol(+)/Kg) & 2.33 & $1.69-8.33$ & 1.07 & $22 \%$ \\
Arena (\%) & 30 & $16-48$ & 6.48 & $16 \%$ \\
Limo (\%) & 45 & $30-62$ & 7.16 & $22 \%$ \\
Arcilla (\%) & 25 & $2-40$ & 5.39 & \\
\hline
\end{tabular}

Tabla 2 - Correlación de Pearson de las propiedades químicas y físicas del suelo. Los valores resaltados presentaron significancia estadística $(\mathrm{P}<0,05)$

\begin{tabular}{lccccccccc}
\hline & $\mathrm{pH}$ & $\mathrm{M}-0$ & $\mathrm{Al}$ & $\mathrm{Ca}$ & $\mathrm{Mg}$ & $\mathrm{K}$ & $\mathrm{CICE}$ & $\mathrm{A}$ & $\mathrm{L}$ \\
\hline $\mathrm{M}-0$ & -0.13 & & & & & & & & \\
$\mathrm{Al}$ & -0.74 & 0.13 & & & & & & & \\
$\mathrm{Ca}$ & 0.49 & 0.01 & -0.28 & & & & & & \\
$\mathrm{Mg}$ & 0.34 & 0.09 & -0.17 & 0.59 & & & & & \\
$\mathrm{~K}$ & 0.30 & 0.10 & -0.23 & 0.31 & 0.37 & & & & \\
$\mathrm{CICE}$ & 0.36 & 0.08 & -0.19 & 0.92 & 0.68 & 0.37 & & & \\
$\mathrm{~A}$ & -0.14 & 0.18 & 0.05 & -0.03 & -0.03 & -0.04 & 0.03 & & \\
$\mathrm{~L}$ & 0.00 & -0.21 & 0.10 & -0.15 & -0.10 & -0.13 & -0.23 & -0.69 & \\
$\mathrm{Ar}$ & 0.16 & 0.06 & -0.18 & 0.24 & 0.17 & 0.21 & 0.27 & -0.28 & -0.50 \\
\hline
\end{tabular}

COMPOSICIÓN FLORÍSTICA

Se registró un total de 2871 individuos pertenecientes a 510 especies arbóreas, agrupadas en 62 familias botánicas para las cinco hectáreas de bosque de tierra firme (Figura 2). Las categorías de abundancias de especies analizadas fueron: especies con abundancia alta (>16 individuos), 36 especies, que corresponden a 1287 individuos. Especies con abundancia media (5 a 16) 108 especies, que corresponden a 916 individuos. Especies con abundancia baja (1 a 4), 366 especies, que corresponden a 668 individuos; ésta última categoría, para efecto de los análisis, se le restaron las especies con sólo un individuo (184 especies), dando como resultado 182 especies que corresponden a 484 individuos. 


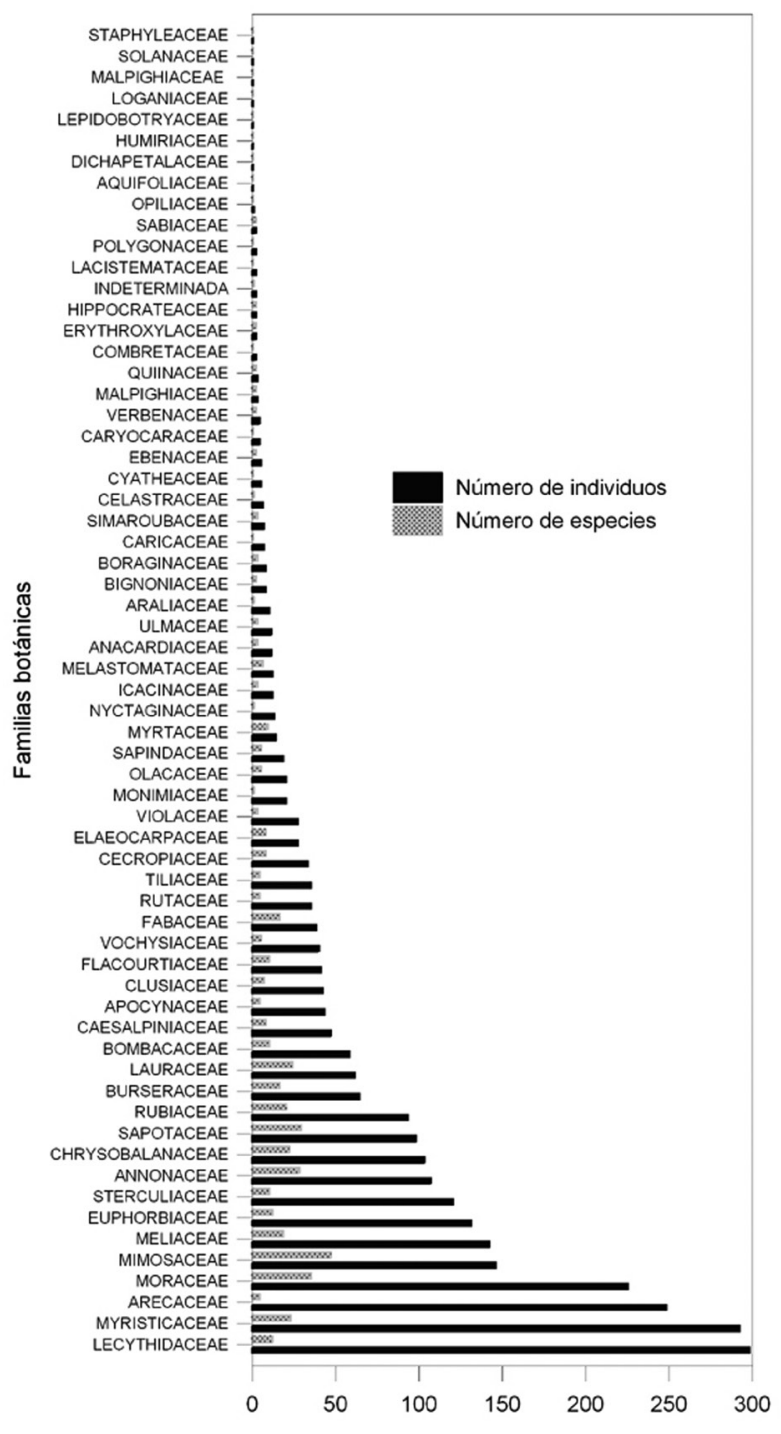

Figura 2 - Abundancia relativa de familias de especies arbóreas en 5-ha de bosque de tierra firme en el Parque Nacional Natural Amacayacu

Las cinco familias más abundantes fueron Lecythidaceae (299 ind), Myristicaceae (293 ind), Arecaceae (249) y Moraceae (226) que en conjunto representan aproximadamente al 50\% del total de individuos. Las especies mas abundantes fueron:

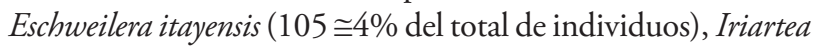
deltoidea (104 ind), Astrocaryum murumuru (100), Otoba parvifolia (92) y Eshweilera coriácea (61).

\section{PATRONES DE VARIABILIDAD FLORÍSTICA}

La diversidad beta, de acuerdo con los resultados del DCA, fue relativamente baja dentro de la parcela. Esta se maximizó cuando se empleó la categoría de especies de abundancia baja, alcanzando aproximadamente 11 desviaciones estándar a lo largo del eje 1 (Tabla 3). Caso contrario, se presentó en la categoría de especies con abundancia alta, donde se minimiza, ya que en el eje 1, resultaron 4.031 desviaciones estándar. En general el diagrama de ordenación separó las unidades muestrales de acuerdo a la disposición de los 125 cuadrantes en las cinco hectáreas (Figura 3).

\section{DISTRIBUCIÓN DE ESPECIES ARBÓREAS Y VARIACIÓN EN SUELOS}

De acuerdo al CCA, las propiedades físico-químicas de los suelos y las variables topográficas que mayor significancia estadística tuvieron al efectuar la selección, ya que presentaron la mayor influencia en la variación de la composición de especies de las unidades muestrales según el grado de explicación y las categoría de abundancia fueron: 1) total de especies: porcentaje de materia orgánica, concentración de aluminio, concentración de calcio, concentración de potasio, fracción de arenas y altitud por unidad muestra; 2) especies con abundancia alta: concentración de aluminio, concentración de calcio, fracción de arenas y altitud por unidad muestral; 3) especies con abundancia media: porcentaje de materia orgánica, CICE, altitud y pendiente por unidad muestral y 4) especies con abundancia baja: fracción de arena, CICE, altitud y pendiente por unidad muestral (Tabla 4).

En general, la partición de la variación total de la composición florística (Figura 3), realizada de acuerdo al total de especies y para las diferentes categorías de abundancia de especies, se tiene que es muy similar entre los dos métodos estudiados (Polinomio y PCNM). Respecto al porcentaje de explicación referido a las variables del ambiente comprende entre el 0 y $6.1 \%$ de explicación. Las variables espaciales explican entre el 1.9 y $35.9 \%$. Respecto a la fracción ambiental espacialmente explicada comprende porcentajes de variación entre 0.2 y $5.1 \%$. Finalmente, el porcentaje de variación

Tabla 3 - Análisis de correspondencia linealizado - DCA

\begin{tabular}{|c|c|c|c|c|}
\hline & \multicolumn{4}{|c|}{ Ejes } \\
\hline & 1 & 2 & 3 & 4 \\
\hline \multicolumn{5}{|c|}{ a. DCA Total de especies } \\
\hline Valores propios & 0.539 & 0.322 & 0.293 & 0.262 \\
\hline Longitud de gradiente & 4.016 & 3.973 & 3.023 & 3.073 \\
\hline Inercia total & \multicolumn{4}{|c|}{16.923} \\
\hline \multicolumn{5}{|c|}{ b. DCA Especies abundancia alta } \\
\hline Valores propios & 0.642 & 0.267 & 0.224 & 0.191 \\
\hline Longitud de gradiente & 3.139 & 2.796 & 2.885 & 2.926 \\
\hline Inercia total & \multicolumn{4}{|c|}{4.461} \\
\hline \multicolumn{5}{|c|}{ c. DCA Especies abundancia media } \\
\hline Valores propios & 0.573 & 0.499 & 0.455 & 0.419 \\
\hline Longitud de gradiente & 8.910 & 4.850 & 4.741 & 4.431 \\
\hline Inercia total & \multicolumn{4}{|c|}{17.277} \\
\hline \multicolumn{5}{|c|}{ d. DCA Especies abundancia baja } \\
\hline Valores propios & 0.860 & 0.833 & 0.773 & 0.731 \\
\hline Longitud de gradiente & 14.515 & 11.067 & 11.298 & 7.477 \\
\hline Inercia total & \multicolumn{4}{|c|}{47.034} \\
\hline
\end{tabular}




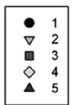

a.

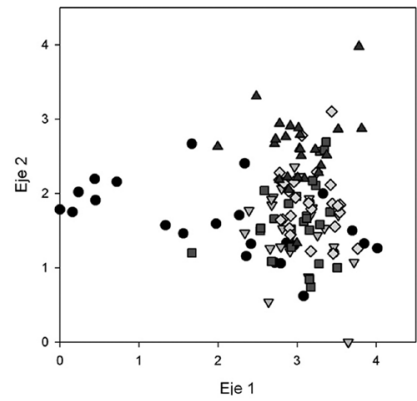

b.

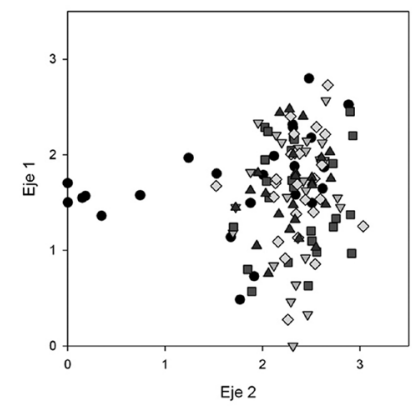

c.

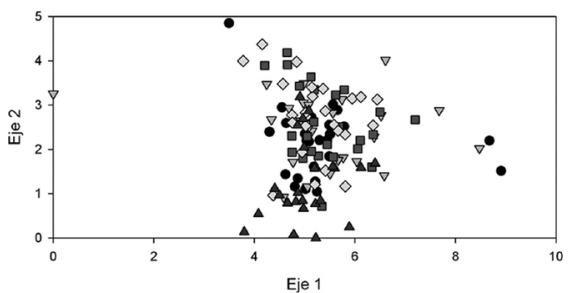

d.

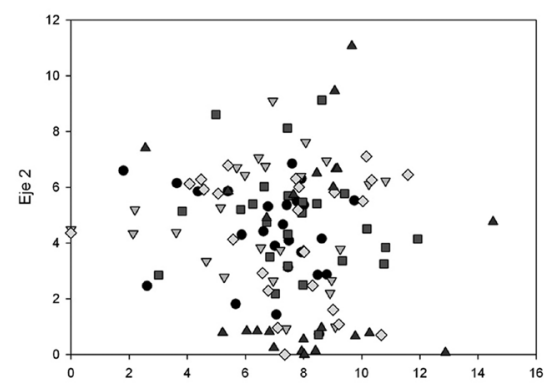

$\square$ No explicad

- Amb+esp
In Puro esp

- Puro amb
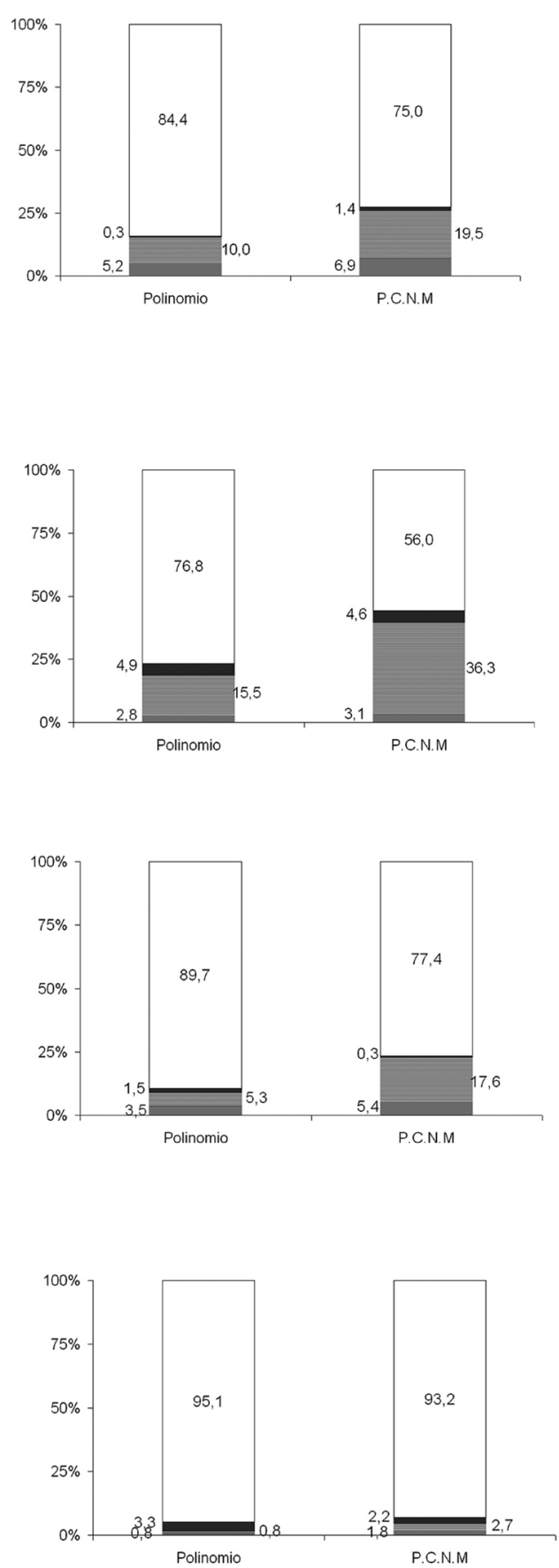

Figura 3 - DCA's y partición de la variación total por categorías de abundancia. En los DCA los números 1-5 corresponden a los cinco cuadrantes de 100x100m. a. Total de especies. b. Especies con abundancia alta. c. Especies con abundancia media. En La partición de la variación total la fracción ambientalmente explicada por el espacio es negativa (-1.5). 
Tabla 4 - Resumen análisis de correspondencia canónico - CCA

\begin{tabular}{|c|c|c|c|c|c|c|c|c|}
\hline & \multicolumn{4}{|c|}{ Ejes Polinomio } & \multicolumn{4}{|c|}{ Ejes PCMN } \\
\hline & 1 & 2 & 3 & 4 & 1 & 2 & 3 & 4 \\
\hline \multicolumn{9}{|c|}{ a. CCA Total de especies } \\
\hline Valores propios & 0.355 & 0.255 & 0,239 & 0,217 & 0.366 & 0.269 & 0.237 & 0.228 \\
\hline $\begin{array}{l}\text { Suma valores } \\
\text { propios }\end{array}$ & \multicolumn{4}{|c|}{16.845} & \multicolumn{4}{|c|}{16.845} \\
\hline $\begin{array}{l}\text { Suma valores } \\
\text { propios canónicos }\end{array}$ & \multicolumn{4}{|c|}{2.625} & \multicolumn{4}{|c|}{4.210} \\
\hline \multicolumn{9}{|c|}{ b. CCA Especies abundancia alta } \\
\hline & 1 & 2 & 3 & 4 & 1 & 2 & 3 & 4 \\
\hline Valores propios & 0.303 & 0.167 & 0.121 & 0.081 & 0.367 & 0.200 & 0.166 & 0.114 \\
\hline $\begin{array}{l}\text { Suma valores } \\
\text { propios }\end{array}$ & \multicolumn{4}{|c|}{3.999} & \multicolumn{4}{|c|}{3.999} \\
\hline $\begin{array}{l}\text { Suma valores } \\
\text { propios canónicos }\end{array}$ & \multicolumn{4}{|c|}{0.926} & \multicolumn{4}{|c|}{1.758} \\
\hline \multicolumn{9}{|c|}{ c. CCA Especies abundancia media } \\
\hline & 1 & 2 & 3 & 4 & 1 & 2 & 3 & 4 \\
\hline Valores propios & 0.342 & 0.306 & 0.229 & 0.179 & 0.381 & 0.342 & 0.276 & 0.239 \\
\hline $\begin{array}{l}\text { Suma valores } \\
\text { propios }\end{array}$ & \multicolumn{4}{|c|}{16.400} & \multicolumn{4}{|c|}{16.400} \\
\hline $\begin{array}{l}\text { Suma valores } \\
\text { propios canónicos }\end{array}$ & \multicolumn{4}{|c|}{1.696} & \multicolumn{4}{|c|}{3.708} \\
\hline \multicolumn{9}{|c|}{ d. CCA Especies abundancia baja } \\
\hline & 1 & 2 & 3 & 4 & 1 & 2 & 3 & 4 \\
\hline Valores propios & 0.536 & 0.521 & 0.437 & 0.431 & 0.545 & 0.542 & 0.477 & 0.64 \\
\hline $\begin{array}{l}\text { Suma valores } \\
\text { propios }\end{array}$ & \multicolumn{4}{|c|}{46.835} & \multicolumn{4}{|c|}{46.835} \\
\hline $\begin{array}{l}\text { Sumavalores } \\
\text { propios canónicos }\end{array}$ & \multicolumn{4}{|c|}{2.294} & \multicolumn{4}{|c|}{3.163} \\
\hline
\end{tabular}

total no explicada, varía entre 56 y $95.1 \%$. Un porcentaje de explicación a resaltar para la categoría de especies con abundancia alta, es el porcentaje de variación explicado por el espacio, debido a las altas densidades que presentan las 36 especies dentro de las 125 unidades muestrales.

\section{DISCUSIÓN}

\section{PATRONES DE DOMINANCIA FLORÍSTICA}

Las familias más dominantes en Amacayacu, tales como Lecythidaceae, Myristicaceae, Arecaceae y Moraceae, que agrupan al $50 \%$ del total de los individuos, coinciden con las reportadas también como dominantes en la parcela del Parque Nacional Yasuní en el Ecuador, y en 14 parcelas de 1-ha en la Reserva de la Biosfera de Manú en el Perú (Condit et al. 2002; Valencia et al. 2004). De igual forma, con la excepción de Eschweilera itayensis, cuatro de las cinco especies más abundantes en este estudio fueron reportadas como especies comunes en áreas aledańas de la Amazonia ecuatoriana y peruana (Pitman et al. 2001). Este patrón apoya la existencia de una alta dominancia regional de relativamente pocos taxones y el carácter generalista de la mayoría de las especies arbóreas en tierra firme dentro de la Amazonia noroccidental (Pitman et al. 1999).

\section{VARIABILIDAD AMBIENTAL REGIONAL Y LOCAL}

La parcela de Amacayacu está localizada sobre depósitos de origen Andino que datan del terciario superior denominados como formación Pebas (Hoorn 1994a,b). Los suelos derivados de esta formación, se caracterizan por tener una mayor fertilidad natural que aquellos derivados del escudo Guyanés los cuales poseen una mayor fracción de arena (Duivenvoorden y Lips 1998). En comparación con la parcela de Yasuní, la variación topográfica es bastante similar, mientras que los suelos parecen ser menos fértiles en Amacayacu. Sin embargo, la interpretación de esta última aseveración debe considerarse con cautela debido a la dificultad de comparar análisis de suelos provenientes de diferentes laboratorios.

A escala local, cuando se consideraron a todas las especies, $\mathrm{Ca}, \mathrm{K}, \mathrm{y} \mathrm{Al}$, fueron elementos importantes determinando la variación florística. Similares resultados se han reportado en otros estudios conducidos en bosques amazónicos, donde $\mathrm{Ca}$ y Al han mostrado una alta correlación con los patrones de distribución de especies a escalas regional y local (Duque $e t$ al. 2001; Tuomisto et al. 2003; Jones et al. 2008). Cuando se consideraron diferentes clases de abundancia, la altitud relativa de la unidad muestral, la cual caracteriza la variación topográfica del terreno, fue siempre significativa dentro de los modelos estudiados. Similares resultados con respecto al efecto proveniente de las catenas de suelos sobre los patrones de distribución de especies a escalas locales fueron encontrados en Yasuní, apoyando la idea de que al menos una proporción de especies responde positivamente a la variación en microhábitat (Valencia et al. 2004; Kraft et al. 2008).

\section{DETERMINANTES DE LA DIVERSIDAD BETA DE ÁRBOLES DEL DOSEL A ESCALA LOCAL}

A escala local, en los bosques de tierra firme del PNN Amacayacu, las diferencias florísticas explicadas a lo largo de la parcela de 5 -ha fueron principalmente asociadas con procesos biológicos como la limitación en dispersión, representada por la fracción espacial. Los factores ambientales relacionados con las características del hábitat, fueron proporcionalmente menos correlacionados con la variación florística, a diferencia de otros estudios desarrollados a escalas similares en bosques del neotrópico (Poulsen et al, 2006, John et al. 2007). Existen dos posibles explicaciones a estas diferencias: En el caso del estudio de Poulsen et al. (2006), donde se consideraron principalmente organismos herbáceos, se podría esperar que estos muestren una mayor especialización edáfica debido a que viven en condiciones de sombra y compiten menos intensamente por luz (Ruokolainen \& Vormisto 2002; Duque et al. 2002). En el segundo caso, John et al. (2007) consideran en su estudio individuos leñosos del sotobosque, los cuales 
parecen responder a cambios en los nichos a lo largo de su ontogenia, lo cual aumentaría el número de especies asociadas con la variación ambiental (Comita et al. 2007). Aún así, es necesario también considerar que la futura inclusión de los elementos leńosos del sotobosque muy posiblemente revele un mayor gregarismo en las especies (Hubbell 1979), y por tanto un incremento en la importancia de la fracción espacial.

La mayor parte de la variación florística, no obstante, no fue explicada por las variables ambientales o espaciales consideradas en el presente estudio (ver Jones et al. 2008). Este resultado sugiere un patrón de distribución de las especies de dosel predominantemente aleatorio. Un patrón de este tipo puede ser explicado por una dispersión de propágulos y un patrón de mortalidad al azar. Procesos denso-dependientes como la mortalidad masiva por el ataque de plagas y enfermedades a cohortes de plántulas y juveniles, aparece como una buena alternativa para explicar este patrón de distribución (Janzen 1970; Harms et al. 2000). Variables no consideradas en este estudio, tales como las características funcionales de las especies, podrían también estar influyendo sobre el alto porcentaje de variación total no explicada, al igual que sobre el bajo porcentaje de variación explicado por la diferenciación de nicho (Kraft et al. 2008). Sin embargo, hasta tanto no se logre una muestra más completa de por lo menos las 25 -ha que tendrá esta parcela incluyendo individuos del sotobosque leñoso $(\mathrm{dap} \geq 1 \mathrm{~cm})$, las conclusiones de este estudio apoyan la idea de que la coexistencia de las especies arbóreas del dosel en estos bosques, está fundamentalmente determinada por procesos asociados con la limitación en dispersión, el azar y la neutralidad (Hubbell 2001).

\section{AUTOCORRELACIÓN ESPACIAL, DIVERSIDAD BETA Y SUBMUESTREO}

En este estudio se confirma que el uso de un polinomio de tercer grado (Borcard et al. 1992), para describir la estructura espacial a escala local o de grano fino, subestimando el efecto real de la autocorrelación (Legendre et al. 2008), y por tanto, la importancia de los procesos biológicos sobre los patrones de distribución de las especies. El uso del PCNM (Borcard y Legendre 2002), en cambio, permite describir de forma más detallada procesos autocorrelativos a un mayor rango de escalas definidas por el grano y dimensión del área de estudio (Jones et al. 2008; Legendre et al. 2008). De esta manera, a diferencia de lo propuesto por Tuomisto y Ruokolainen (2006), se demuestra que la predicción proveniente de la teoría neutral (Hubbell 2001), acerca de la presencia de autocorrelación positiva en la composición florística de las comunidades debido a la limitación en dispersión, es factible y viable usando datos brutos aún a escalas pequeñas.

Con el fin de explorar el efecto del submuestreo sobre los resultados obtenidos, nosotros dividimos los datos totales por categorías de abundancia. Entre las lecciones aprendidas se destacan: 1) muestras basadas en especies más abundantes van a evidenciar de manera más precisa el efecto de la limitación en dispersión. Nótese que en este caso, usando ambos métodos, fue cuando se maximizó el total de variación explicada de los datos florísticos, básicamente por un incremento en la correlación con la fracción espacial (Figura 3b); 2) a diferencia de lo propuesto por algunos autores (e.g., Preston 1962), el uso de especies con abundancia intermedia mostró una disminución en el porcentaje de variación explicada por la fracción ambiental; el uso del PCNM muestra que este tipo de especies están especialmente regidas en cuanto a su patrón de distribución por procesos estocásticos o autocorrelacionados y 3) el uso de especies pobremente representadas en la muestra (2-4 individuos), promueve la disminución de la variación total explicada de ambas fracciones. El aumento del área total de muestreo con seguridad dará nuevas luces para entender mejor la dependencia del patrón de las especies raras con los suelos, la topografía y/o la limitación en dispersión.

\section{AGRADECIMIENTOS}

Los autores del presente estudio extendemos nuestro especial agradecimiento a la Dirección de Parques Nacionales Naturales de Colombia, y muy en especial a los miembros del PNN Amacayacu. Deseamos agradecer muy en especial a los dos directores de turno Jaime Alberto Celis y Alexander Alfonso Segura. A todos los colaboradores de campo y en general a toda la comunidad de Palmeras. A aquellos especialistas que colaboraron con la determinación del material botánico. El presente trabajo fue posible gracias a la financiación recibida de COLCIENCIAS (CT 1118333-18676) y el Center for Tropical Forest Science (CTFS; subcontrato \# 08-440-0000137735).

\section{BIBLIOGRAFIA CITADA}

Alonso, D.; Ostling, A.; Etienne, R.S. 2008. The implicit assumption of symmetry and the species abundance distribution. Ecology Letters, 11: 93-105.

Borcard, D.; Legendre, P.; Drapeau, P. 1992. Partialing out the spatial component of ecological variation. Ecology, 73: 1045-1055.

Borcard, D.; Legendre, P. 2002. All-scale spatial analysis of ecological data by means of principal coodinates of neighbour matrices. Ecological Modelling, 153: 51-68.

Brown, J.H.; Mehlman, D.W.; Stevens, G.C. 1995. Spatial variation in abundance. Ecology, 76: 2020-2043.

Comita, L.; Condit, R.; Hubbell, S.P. 2007. Developmental changes in habitat associations of tropical trees. Journal of Ecology, 95: 482-492.

Condit, R. 1998. Tropical forest census plots. Springer-Verlag, Berlin.211pp.

Condit, R.; Ashton, P.S.; Baker, P.; Bunyavejchewin, S.; Gunatilleke, S.; Gunatilleke, N.; Hubbell, S.P.; Foster, R.B., Itoh, A.; 
LaFrankie, J.V.; Lee, H.S.; Losos, E.; Manokaran, N.; Sukumar, R.; Yamakura, T. 2000. Spatial patterns in the distribution of tropical tree species. Science, 288: 1414-1418.

Condit R.; Pitman, N.; Leigh, E.; Chave, J.; Terborgh, J.; Foster R.B.; Nuñez, P.; Aguilar, S.; Valencia, R.; Villa, G.; Muller-Laundau, H.; Losos, E.; Hubbell, S. 2002. Beta-diversity in tropical forest trees. Science, 295: 666-668.

Duque, A.; Sánchez, M.; Cavelier, J.; Duivenvoorden, J.F.; Miraña, P.; Miraña, J.; Matapi, A. 2001. Relación bosque-ambiente en el Medio Caquetá, Amazonia Colombiana: p. 99-130. In: Duivenvoorden, J.F.; Baslev, H.; Cavelier, J.; Grandez, C.; Tuomisto, H.; Valencia, R. (Eds.). Evaluación de recursos vegetales no maderables en la Amazonia Noroccidental. IBED, Universiteit van Ámsterdam, Ámsterdam, Holanda. 486 pp.

Duque, A.; Sánchez, M.; Cavelier, J.; Duivenvoorden, J.F. 2002. Different floristic patterns of woody understory and canopy plants in Colombian Amazonia. Journal of Tropical Ecology, 18: 499-525.

Duque, A.; Cavelier, J.; Posada, A. 2003. Strategies of tree occupation at a local scale in terra firme Forests in the Colombian Amazon. Biotropica, 35: 20-27.

Duivenoorden, J.F.; Lips, J.M. 1995. A land-ecological study of soils, vegetation, and plant diversity in Colombian Amazonia. Tropenbos series 12. Fundación Tropenbos, Wageningen, Holanda. 438 p.

Duivenvoorden, J.F.; Duque, A. In press. Diversity and composition of north-western Amazonian forest in a spatio-dynamic geoecological context. In: Hoorn, C.; Wesselingh, F.; Vonhof, H. (Eds.). Neogene history of Western Amazonia and its significance for modern biodiversity. Blackwell.

Gentry, A.H. 1988. Changes in plant community diversity and floristic composition on environmental and geographic gradients. Annals of the Missouri Botanical Garden, 75: 1-34.

Harms, K.E.; Wright, S.J.; Calderón, O.; Hernández, A.; Herre, E.A. 2000. Pervasive density-dependent recruitment enhances seedling diversity in a tropical forest. Nature, 404: 493-495.

Harms, K.E.; Condit, R.; Hubbell, S.P.; Foster, R.B. 2001. Habitat associations of trees and shrubs in a neotropical forest. Journal of Ecology, 89: 947-959.

He F.; Legendre P.; LaFrankie J. 1996. Spatial pattern of diversity in a tropical rain forest in Malaysia. Journal of Biogeography, 23: $57-74$.

He, F.; LaFrankie, J.; Song B. 2002. Scale dependence of tree abundance and richness in a tropical rain forest, Malaysia. Landscape Ecology, 17: 559-568.

He, F.; Hu, X.-S. 2005. Hubbell's fundamental biodiversity parameter and the Simpson diversity index. Ecology Letters, 8: 386-390.

Holdridge, L.R. 1979. Ecología basada en zonas de vida. Editorial IICA-San José. Costa Rica. 197 pp.

Hoorn, C. 1994.Fluvial paleoenvironments in the intercratonic Amazonas Basin (Early Miocene to EARLY Middle Miocene, Colombia). Palaeogeography; Palaeoclimatology, Palaeoecology, 109: 1-55.

Hoorn, C. 1994. An environmental reconstrucción of the palaeoamazon river system (Middle to late Miocene, NW Amazonia).
Palaeogeography; Palaeoclimatology, Palaeoecology, 112: 187238.

Hubbell, S.P. 1979. Tree dispersion, abundance and diversity in a tropical dry forest. Science, 203: 1299-1309.

Hubbell, S.P. 2001. The unified neutral theory of biodiversity and biogeography. Monographs in population biology 32. Princeton University Press. USA. 375pp.

Hubbell, S.P. 2005. Neutral theory in community ecology and the hypothesis of functional equivalence. Functional Ecology, 19: 166-172.

Hutchinson, G.E. 1959. Homage to Santa Rosalia or why are there so many kinds of animals? The American Naturalist, 93: 145-159.

Janzen, D.H. 1970. Herbivores and the number of tree species in tropical forests. The American Naturalist, 104: 501-528.

John; R.; Dalling, J.W.; Harms, K.E.; Yavitt, J.B.; Stallard, R.F.; Mirabello, M.; Hubbell, S.P.; Valencia, R.; Navarrete, H.; Vallejo, M; Foster, R.B. 2007. Soil nutrients influence spatial distributions of tropical tree species. Proceedings of National Academy of Sciences, 104: 864-869.

Jones, M.M.; Tuomisto, H.; Borcard, D.; Legendre, P.; Clark, D.B.; Olivas, P.C. 2008. Explainig variation in tropical plant community composition: influence of environtmental and spatial data quality. Oecologia, 155: 593-604.

Jongman, R.G.H.; ter Braak; C.J.F.; van Tongeren, O.F.R. 1987. Data analysis in community and landscape ecology. Centre for Agricultural Publishing an Documentation (PUDOC), Wageningen, Holanda. 324 pp.

Kraft, N.J.B.; Valencia, R.; Ackerly, D.D. 2008. Functional traits and niche-based tree community assembly in an Amazonian forest. Science, 322: 580-582.

Legendre, P.; Borcard, D.; Peres-Neto, P.R. 2008. Analyzing or explainig beta diversity? Comment. Ecology, 89: 3238-3244.

Matteucci, S.D.;.Colma, A. 1982. Metodología para el estudio de la vegetación. Secretaria General de la Organización de los Estados Americanos. Programa Regional de Desarrollo Científico y Tecnológico, Washington D.C., USA. 168 pp.

McGill, B.J.; Etienne, R.S.; Gray, J.S.; Alonso, D.; Anderson, M.J.; Benecha, H.K.; Dornelas, M.; Enquist, B.J.; Green, J.L.; He, F.; Hurlbert, A.H.; Magurran, A.E.; Marquet, P.A.; Maurer, B.A.; Ostling, A.; Soykan, C.U.; Ugland, K.I.; White, E.P. 2007. Species abundance distributions: moving beyond single prediction theories to integration within an ecological framework. Ecology Letters, 10: 995-1015.

Pitman, N.C.A.; Terborgh, J.; Silman, M.R.; Nuñez, P. 1999. Tree species distributions in an upper Amazonian forest. Ecology, 80: 2651-2661

Pitman, N.C.A.; Terborgh, J.; Silman, M.R.; Nuñez, P.; Neill, D.A.; Cerón, C.E.; Palacios, W.A.; Aulestia, M. 2001. Dominance and distribution of tree species in upper Amazonian tierra firme forest. Ecology, 82: 2101-2117.

Poulsen, A.D.; Tuomisto, H.; Baslev, H. 2006. Edaphic and Floristic Variation a 1-ha Plot of Lowland Amazonian Rain Forest. Biotropica, 38: 468-478. 
Preston, F.W. 1948. The commonness, and rarity, of species. Ecology, 29: 254-283.

Preston, F.W. 1962. The Canonical Distribution of Commonness and Rarity: Part I. Ecology, 43: 185-215.

Pyke, C.R.; Condit, R.; Aguilar, S.; Lao, S. 2001. Floristic composition across a climatic gradient in a neotropical lowland forest. Journal of Vegetation Science, 12: 553-566.

Rees, M.; Condit, R.; Crawley, M.; Pacala; S.; Tilman, D. 2001. Long-term studies of vegetation dynamics. Science, 293: 650655.

Ruokolainen, K.; Vormisto, J. 2002. The most widespread Amazonian palms tend to be tall and habitat generalist. Journal of Ttropical Ecology, 23: 13-25.

Svenning, J.-C. 1999. Microhabitat specialization en a species-rich palm community in Amazonian Ecuador. Journal of Ecology, 87: 55-65.

ter Braak, C.J.F; Smilauer, P. 2002. CANOCO Reference manual and Canodraw for Windows user's guide: Software for Canonical Community Ordination (version 4.5). Microcomputer Power, Ithaca, NY, USA. 500 pp.
Tilman, D.; Pacala, S. 1993. The maintenance of species diversity in plant communities. Species Diversity in Ecological Communities. University of Chicago Press, Chicago.USA.p. 13-25.

Tuomisto, H.; Ruokolainen K.; Kalliola, R.; Linna, A.; Danjoy, W.; Rodriguez, Z. 1995. Dissecting Amazonian biodiversity. Science, 269: 63-66.

Tuomisto H.; Ruokolainen K.; Yli-Halla M. 2003. Dispersal, environment, and floristic variation of western amazonian forests. Science, 299: 241-244.

Valencia, R.; Foster, R.B.; Villa, G.; Condit, R.; Svenning, J.E.; Hernández, C.; Romoleroux, K.; Losos, E.; Magard, E.; Balslev, H. 2004. Tree species distributions and local habitat variation in the Amazon: large forest plot in eastern Ecuador. Journal of Ecology, 92: 214-229.

Zillio, T.; Condit, R. 2007. The impact of neutrality, niche differentiation and species input on diversity and abundance distributions. Oikos, 116: 931-940.

Recebido em 22/10/2009

Aceito em 03/11/2009 